\title{
Journal of Mining and Earth Sciences
}

Website: http://jmes.humg.edu.vn

\section{Detect and process outliers for temperature data at $3 \mathrm{~h}$ monitoring stations in Vietnam}

\section{Nam Van Dang ${ }^{1,}{ }^{*}$, Oanh Thi Nong ${ }^{1}$, Hoai Xuan Nguyen ${ }^{2}$, Manh Van Ngo ${ }^{3}$, Hien Thi} Nguyen ${ }^{4}$

${ }^{1}$ Faculty of Information Technology, Hanoi University of Mining and Geology, Vietnam

${ }^{2}$ AI Academy Vietnam, Vietnam

${ }^{3}$ Center for Hydro - Meteorological Data and Information, Vietnam

${ }^{4}$ Falculty of Information Technology, Technical University, Vietnam

ARTICLE INFO

Article history:

Received $11^{\text {th }}$ Nov. 2019

Revised 06 ${ }^{\text {th }}$ Mar. 2020

Accepted $28^{\text {th }}$ Feb. 2020

Keywords:

Anomalies,

Box-plot,

Outliers,

Z-Score.
ABSTRACT

Data preparation is a compulsory process in any data science project. Many research have shown that it constitutes $80 \%$ of the time, effort and resources of a data science project. Depending on the particular project and data type, Data preparation step may required different methods/steps. Detecting and processing outlier data is one of the important preprocessing steps in data preparation, especially for time series data. This paper reviews two methods for detecting outliers for low dimensional data, namely Z - Score and Box - plot charts. We also present results of experiments which applied these methods for temperature data collected from 43 monitoring stations in 3 - hour in Vietnam over the last 6 years from 01/01/2014 to 31/12/2019.

Copyright (C) 2020 Hanoi University of Mining and Geology. All rights reserved.

${ }^{*}$ Corresponding author

E-mail: dangvannam@humg.edu.vn

DOI: 10.46326/JMES.2020.61(1).15 


\title{
Tạp chí Khoa học Kỹ thuật Mỏ - Địa chất
}

Trang điện tử: http://tapchi.humg.edu.vn

\section{Phát hiện và xử lý ngoại lai cho dữ liệu nhiệt độ tại các trạm quan trắc 3h của Việt Nam}

\author{
Đặng Văn Nam ${ }^{1}{ }^{*}$, Nông Thị Oanh ${ }^{1}$, Nguyễn Xuân Hoài ${ }^{2}$, Ngô Văn Mạnh ${ }^{3}$, Nguyễn \\ Thị Hiền ${ }^{4}$ \\ ${ }^{1}$ Khoa Công nghê Thông tin, Trường Đại học Mỏ - Địa chất, Việt Nam \\ 2 Viện Trí tuệ nhân tạo, Việt Nam \\ 3 Trung tâm Thông tin và Dũ liệu khí tượng thủy văn, Việt Nam \\ ${ }^{4}$ Học viện Kỹ thuật quân sự, Việt Nam
}

\section{THÔNG TIN BÀI BÁO TÓM TẮT}

\section{Quá trình:}

Nhận bài $15 / 11 / 2019$

Sửa xong 06/01/2020

Chấp nhận đăng 28/02/2020

Tù̀ khóa:

Anomalies,

Box - plot,

Outliers,

Z - Score.
Trong bất kỳ một dự án khoa hoc dũ liẹu nào thì chuẩn bi dũ liệu (Data preparation) là công đoạn bắt buộc và không thể thiếu. Kết quả của nhiều nghiên cúu đã chỉ ra rằng, chuẩn bị dũ liệu là công đoạn chiếm tới 80\% thời gian, công sức và nguồn lực của một dự án khoa học dữ liệu. Chuẩn bị dũ liệu bao gồm rất nhiều bước xủ lý, với nhiều nghiệp vu khác nhau và phu thuộc vào tùng bài toán, tùng loại dũ liệu cụ thể. Phát hiện và xử lý dữ liẹu ngoại lai (Outliers) là một trong nhũng bước tiền xử lý quan trọng, đặc biệt là các dũ liệu số dạng chuỗi thời gian (Time series) (Hermine N. Akouemo et al., 2014). Trong nội dung của bài báo này, tác giả nghiên cứu hai phương pháp hiệu quả đang được sử dụng để phát hiện ngoại lai cho dũ liệu có số chiều thấp là $Z$ - Score và biểu đồ Box - plot, cũng như các phương pháp để xử lý dũ liệu ngoại lai nói chung. Sau đó tiến hành thực nghiệm, áp dụng những phương pháp phát hiện và xử lý này cho dũ liệu nhiệt độ thu thập được từ 43 trạm quan trắc 3 h của Việt Nam trong giai đoạn 6 năm gần đầy tù̀ năm 2014 đến năm 2019.

\section{Mở đầu}

Dữ liệu khí tượng thủy văn trong đó có dữ liệu nhiệt độ, được thu thập, xử lý và lưu trữ tại cơ sở dữ liệu của Trung tâm thông tin và Dư liệu khí tượng thủy văn. Đây là dữ liệu dạng chuỗi thời gian được thu thập định kỳ theo từng khoảng thời

*Tác giả liên hệ

E - mail: dangvannam@humg.edu.vn DOI: 10.46326/JMES.2020.61(1).15 gian nhất định (3 giờ hoặc 6 giờ) tùy thuộc vào từng trạm cụ thể. Hiện tại ở Việt Nam, có 43 trạm quan trắc dữ liệu với tần suất 3 giờ một lần, chi tiết các trạm quan trắc và dữ liệu được trình bày cụ thể trong phần 2 của bài báo. Quá trình đo đạc, xử lý, tổng hợp, truyền và lưu trữ dữ liệu quan trắc từ các trạm bị ảnh hưởng bởi các yếu tố chủ quan và khách quan dẫn đến mất dữ liệu và/hoặc tác động đến độ chính xác của dữ liệu. Do đó, yêu cầu bắt buộc là dữ liệu cần phải được chuẩn hóa (Data preparation) trước khi sử dụng cho bất kỳ mục đích gì. 
Theo Davy Cielen et al. (2016) đã chỉ ra rằng, chuẩn bị dữ liệu được đánh giá là khâu chiếm nhiều thời gian, công sức và nguồn lực nhất của bất kỳ một dự án khoa học dữ liệu nào. Các kết quả nghiên cứu cho thấy $80 \%$ thời gian, công sức và nguồn lực của một dự án khoa học dữ liệu là cho việc này. Chuẩn bị dữ liệu bao gồm rất nhiều thao tác, nghiệp vụ, kỹ thuật và yêu cầu khác nhau, phụ thuộc vào từng loại dữ liệu và từng dự án cụ thể. Tuy nhiên, chúng ta có thể tổng hợp vào ba nhóm thao tác chính: Làm sạch dữ liệu (Data cleansing); Chuyển đổi dữ liệu (Data transformation) và tích hợp dữ liệu (Combining data).

Khi nghiên cứu và làm việc với dữ liệu khí tượng thủy văn nói chung, dữ liệu nhiệt độ nói riêng tác giả thấy rằng, việc chuẩn hóa dữ liệu cho dữ liệu nhiệt độ tập trung chủ yếu vào 4 vấn đề chính dưới đây:

- Kết hợp và sắp xếp dữ liệu quan trắc theo chuỗi thời gian và theo vị trí địa lý của các trạm.

- Phát hiện và xử lý các dữ liệu ngoại lai (Outliers) trong tập dữ liệu quan trắc.

- Phát hiện và xử lý các dữ liệu thiếu (Missing data) trong tập dữ liệu quan trắc.

- Chuyển đổi, định dạng và xuất dữ liệu đã xử lý để lưu trữ theo yêu cầu.

Các điểm dữ liệu ngoại lai hay còn được gọi là các dữ liệu bất thường (Anomalies) có ảnh hưởng lớn đến độ chính xác của các mô hình dự đoán. Phát hiện và xử lý ngoại lai là thao tác quan trọng trong quá trình làm sạch dữ liệu. Việc phát hiện ngoại lai giúp phát hiện ra những điểm dữ liệu không phù hợp hay bất thường hơn so với phần còn lại của tập dữ liệu (C. Aggarwal, 2017).

Phát hiện ngoại lai không chỉ được ứng dụng trong việc làm sạch dữ liệu mà nó còn được ứng dụng vào nhiều bài toán thực tế như: Phát hiện lỗi (fraud detection); giám sát (surveillance); chuẩn đoán (diagnosis); dự đoán bảo trì (predictive maintaence),... Tuy nhiên, việc phát hiện các điểm dữ liệu ngoại lai không phải là một công việc đơn giản, nó yêu cầu phải có những hiểu biết sâu sắc về tập dữ liệu, cũng như nắm vững các phương pháp hiệu quả để thực hiện việc này.

Trong nội dung của bài báo, nhóm tác giả tập trung giải quyết một trong số bốn vấn đề chính đã chỉ ra ở trên, đó là nhiên cứu các phương pháp phát hiện và xử lý ngoại lai, trên cơ sở đó áp dụng các phương pháp này vào việc xử lý ngoại lai cho dữ liệu nhiệt độ thu thập được tại toàn bộ 43 trạm quan trắc 3 giờ của Việt Nam trong khoảng thời gian 6 năm gần đây, từ ngày 1 tháng 1 năm 2014 tới hết ngày 31 tháng 12 năm 2019. Toàn bộ dữ liệu sử dụng trong bài báo đều là dữ liệu thực tế được cung cấp bởi Trung tâm thông tin và dữ liệu khí tượng thủy văn.

\section{Dữ liệu nhiệt độ tại các trạm quan trắc $3 h$}

Trạm quan trắc khí tượng thủy văn được lắp đặt tại các vị trí khác nhau để thực hiện đo các thông số khí tượng như: Nhiệt độ, tốc độ gió, hướng gió, lượng mưa, độ ẩm,... Các trạm này định kỳ sau một khoảng thời gian cố định được thiết lập thực hiện việc đo các thông số này. Với các trạm quan trắc 3h sẽ thực hiện thu thập dữ liệu 8 lần mỗi ngày, mỗi lần cách nhau 3 giờ tại các thời điểm 00h, 03h, 06h, 09h, 12h, 15h, 18h, 21h theo giờ GMT, tương ứng với $01 \mathrm{~h}, 04 \mathrm{~h}, 07 \mathrm{~h}, 10 \mathrm{~h}, 13 \mathrm{~h}, 16 \mathrm{~h}$, $19 h, 22 h$ giờ Việt Nam. Ở nước ta hiện nay, có tổng số 43 trạm quan trắc khí tượng thủy văn với thời gian quan trắc là $3 \mathrm{~h}$ một lần. Danh sách 43 trạm này được cho trong Bảng 1 , vị trí đặt các trạm được thể hiện trong Hình 1.

Dữ liệu khí tượng thủy văn nói chung, dữ liệu nhiệt độ nói riêng, sau khi được đo đạc từ các trạm quan trắc sẽ được gửi về Trung tâm thông tin và dữ liệu khí tượng thủy văn.

Dữ liệu được lưu trũ trong cơ sở dữ liệu MongoDB, tiến hành kết nối tới máy chủ cơ sở dữ liệu và truy xuất thông số nhiệt độ của 43 trạm trong khoảng thời gian từ $01 \mathrm{~h}$ ngày $01 / 01 / 2014$ tới $22 \mathrm{~h}$ ngày $31 / 12 / 2019$.

Các dữ liệu nhiệt độ sau đó được lưu trữ ra tệp định dạng.CSV (Comma - separated values) có tên Data_Temp43_Original.csv (Hình 2) để thuận tiện cho việc xử lý các bước tiếp theo. Cột đầu tiên trong tệp có tên "TimeVN" cho biết thời điểm quan trắc dữ liệu, các cột còn lại (tiêu đề mỗi cột tương ứng với mã trạm quan trắc) là dữ liệu nhiệt độ của từng trạm ứng với mốc thời gian của cột "TimeVN". Đây là tệp dữ liệu gốc (dữ liệu thô - Raw dataset) được tổng hợp khi các trạm gửi về, quá trình thu thập dữ liệu, truyền nhận và lưu trữ có thể do các nguyên nhân chủ quan và khách quan dẫn đến dữ liệu có thể bị mất, bị sai lệch,... Do đó, trước khi sử dụng các số liệu này cần phải được xử lý. 
Bảng 1. Danh sách 43 trạm quan trắc 3h của Việt Nam.

\begin{tabular}{|c|c|c|c|c|c|}
\hline STT & Mã trạm & Tên quốc tế & Tên Việt Nam & Trạm đảo & Tỉnh/Thành phố \\
\hline 1 & 48800 & MUONG LAY & Mường Lay & & Điện Biên \\
\hline 2 & 48811 & DIEN BIEN & Điện Biên & & Điện Biên \\
\hline 3 & 48806 & SON LA & Sơn La & & Sơn La \\
\hline 4 & 48818 & HOA BINH & Hòa Bình & & Hòa Bình \\
\hline 5 & 48803 & LAO CAI & Lào Cai & & Lào Cai \\
\hline 6 & 48805 & HA GIANG & Hà Giang & & Hà Giang \\
\hline 7 & 48812 & TUYEN QUANG & Tuyên Quang & & Tuyên Quang \\
\hline 8 & 48808 & CAO BANG & Cao Bằng & & Cao Bằng \\
\hline 9 & 48830 & LANG SON & Lạng Sơn & & Lạng Sơn \\
\hline 10 & 48838 & MONG CAI & Móng Cái & & Quảng Ninh \\
\hline 11 & 48837 & TIEN YEN & Tiên Yên & & Quảng Ninh \\
\hline 12 & 48833 & BAI CHAY & Bãi Cháy & & Quảng Ninh \\
\hline 13 & 48826 & PHU LIEN & Phù Liễn & & Hải Phòng \\
\hline 14 & 48839 & BACH LONG VI & Bạch Long Vĩ & $\mathrm{X}$ & Hải Phòng \\
\hline 15 & 48825 & HA DONG & Hà Đông & & Hà Nội \\
\hline 16 & 48823 & NAM DINH & Nam Định & & Nam Định \\
\hline 17 & 48842 & HOI XUAN & Hồi Xuân & & Thanh Hóa \\
\hline 18 & 48840 & THANH HOA & Thanh Hóa & & Thanh Hóa \\
\hline 19 & 48845 & VINH & Vinh & & Nghệ An \\
\hline 20 & 48846 & HA TINH & Hà Tĩnh & & Hà Tĩnh \\
\hline 21 & $48 / 86$ & KY ANH & Kỳ Anh & & Hà Tĩnh \\
\hline 22 & 48848 & DONG HOI & Đồng Hới & & Quảng Bình \\
\hline 23 & 48852 & HUE & Huế & & Thừa Thiên Huế \\
\hline 24 & 48860 & HOANG SA & Hoàng Sa & & Đà Nẵng \\
\hline 25 & 48855 & DA NANG & Đà Nẵng & & Đà Nẵng \\
\hline 26 & 48863 & QUANG NGAI & Quảng Ngãi & & Quảng Ngãi \\
\hline 27 & $48 / 96$ & HOAI NHON & Hoài Nhơn & & Bình Định \\
\hline 28 & 48870 & QUY NHON & Quy Nhơn & & Bình Định \\
\hline 29 & 48873 & TUY HOA & Tuy Hòa & & Phú Yên \\
\hline 30 & 48877 & NHA TRANG & Nha Trang & & Khánh Hòa \\
\hline 31 & 48920 & TRUONG SA & Trường Sa & $\mathrm{X}$ & Khánh Hòa \\
\hline 32 & 48890 & PHAN RANG & Phan Rang & & Ninh Thuận \\
\hline 33 & 48887 & PHAN THIET & Phan Thiết & & Bình Thuận \\
\hline 34 & 48889 & PHU QUY & Phú Quý & $\mathrm{X}$ & Bình Thuận \\
\hline 35 & 48866 & PLEIKU & Pleiku & & Gia Lai \\
\hline 36 & 48875 & BUON MA THUAT & Buôn Ma Thuật & & Đắc Lắc \\
\hline 37 & 48894 & NHA BE & Nhà Bè & & Hồ Chí Minh \\
\hline 38 & 48903 & VUNG TAU & Vũng Tàu & & Bà Rịa - Vũng Tàu \\
\hline 39 & 48918 & CON DAO & Côn Đảo & $\mathrm{X}$ & Bà Rịa - Vũng Tàu \\
\hline 40 & 48910 & CAN THO & Cần Thơ & & Cần Thơ \\
\hline 41 & 48917 & PHU QUOC & Phú Quốc & $\mathrm{X}$ & Kiên Giang \\
\hline 42 & 48907 & RACH GIA & Rạch Giá & & Kiên Giang \\
\hline 43 & 48914 & CA MAU & Cà Mau & & Cà Mau \\
\hline
\end{tabular}




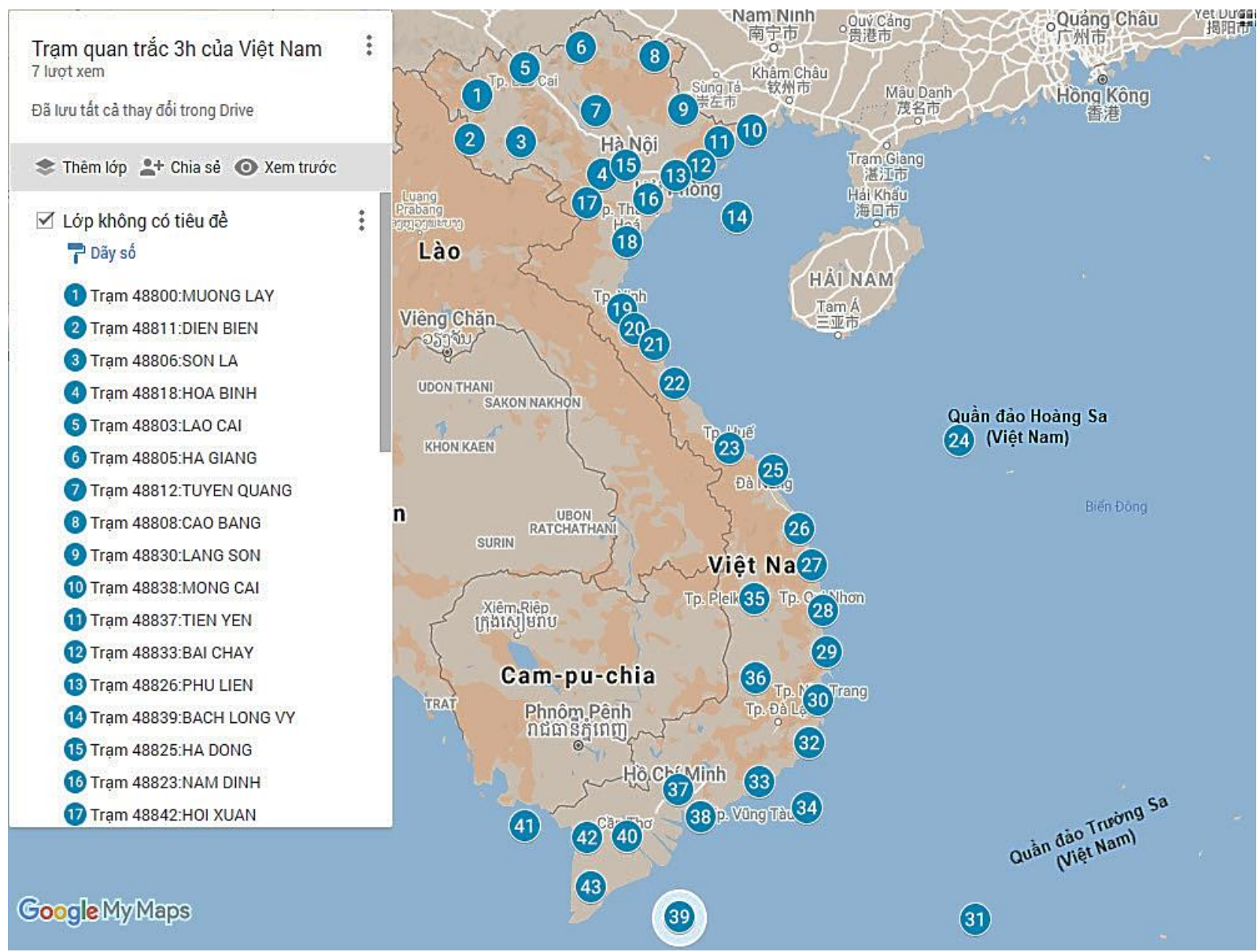

Hình 1. Vị trí các trạm quan trắc 3h trên bản đồ Google Maps.

\begin{tabular}{|c|c|c|c|c|c|c|c|c|c|c|c|c|c|c|}
\hline$\Delta$ & A & B & C & D & $\mathrm{E}$ & F & $\mathbf{G}$ & $\mathrm{H}$ & I & $\mathbf{J}$ & $\mathrm{K}$ & $\mathrm{L}$ & \multirow{2}{*}{\multicolumn{2}{|c|}{$\begin{array}{ll}N & \\
48826\end{array}$}} \\
\hline 1 & TimeVN & 48500 & 48511 & 48506 & 48818 & 48803 & 48805 & 48512 & 48308 & 48830 & 48838 & 48837 & & \\
\hline 2 & 2014-01-01 1:00 & 15.4 & 14 & 7.6 & 10.2 & 10.9 & 10.1 & 10.4 & 6.7 & 5.3 & 8.8 & 9.2 & 10.8 & 13.8 \\
\hline 3 & 2014-01-01 4:00 & 15 & 13.8 & 8.6 & 9.4 & 11.2 & 9.1 & 9.2 & 6 & 3.8 & 8.1 & 8.2 & 10 & 13.4 \\
\hline 4 & 2014-01-01 7:00 & 15.1 & 13.4 & 9.4 & 8.6 & 11.6 & 8.6 & 8.4 & 5.6 & 3.3 & 7 & 7.2 & 9.6 & 13.2 \\
\hline 5 & $2014-01-01 \quad 10=00$ & 17.4 & 15 & 11.2 & 16.1 & 13.6 & 13.7 & 10.8 & 7.3 & 11.3 & 17.8 & 15.5 & 14.8 & 16.4 \\
\hline 6 & 2014-01-01 13:00 & 21.4 & 19 & 14.7 & 22.4 & 19.4 & 20.7 & 20.2 & 18.6 & 20.1 & 20.7 & 19.7 & 19.6 & 20.4 \\
\hline 7 & 2014-01-01 16:00 & 21.2 & 21.4 & 17 & 22.2 & 21 & 21.1 & 21.1 & 21.2 & 20.6 & 18.2 & 19.9 & 18.9 & 20.4 \\
\hline 8 & 2014-01-01 19:00 & 17.3 & 17.5 & 13 & 16.7 & 15.8 & 16.2 & 15.9 & 13.5 & 12.7 & 12.2 & 12.6 & 16.1 & 16.1 \\
\hline 9 & 2014-01-01 22:00 & 15.1 & 14.1 & 10.4 & 12.9 & 12.5 & 11.8 & 13.2 & 9.6 & 9.4 & 9.9 & 9 & 13.3 & 15.4 \\
\hline 10 & 2014-01-02 1:00 & 14.6 & 12.3 & 9.4 & 11.3 & 11 & 10.1 & 11.4 & 7.9 & 7.7 & 8.6 & 8.6 & 11.6 & 14.6 \\
\hline 11 & 2014-01-02 4:00 & 13.6 & 11.8 & 8.6 & 10.2 & 9.6 & 9.2 & 10.6 & 7 & 6.4 & 8.4 & 7.6 & 10.9 & 14.3 \\
\hline 12 & 2014-01-02 7:00 & 13.2 & 11.8 & 8 & 10 & 9 & 9.9 & 9.1 & 6.4 & 5.9 & 7.7 & 7.6 & 10.7 & 13.8 \\
\hline 13 & 2014-01-02 10:00 & 15.2 & 15.2 & 17.3 & 16.4 & 12.4 & 15.2 & 12.5 & 8.7 & 17.4 & 18.8 & 16.2 & 15 & 18.2 \\
\hline 14 & 2014-01-02 13:00 & 21.1 & 20.2 & 22 & 22.6 & 20.4 & 21.6 & 20.5 & 20.9 & 20.3 & 20.8 & 22.6 & 20.4 & 21.6 \\
\hline 15 & 2014-01-02 16:00 & 25.6 & 24.6 & 23.4 & 24.2 & 23 & 21.8 & 22.3 & 23.8 & 21.3 & 19.4 & 21.4 & 19 & 20.8 \\
\hline 16 & $2014-01-02$ 19:00 & 19.6 & 18.6 & 16.4 & 17.8 & & & & & & & & & \\
\hline 17 & $2014-01-0222: 00$ & 17.6 & 14.3 & 13.5 & 15 & & & & & & & & & \\
\hline 18 & 2014-01-03 1:00 & 15.1 & 14.1 & 11.5 & 13.7 & & & & & & & & & \\
\hline 19 & 2014-01-03 4:00 & 14.7 & 13.7 & 10.6 & 14.5 & & & & & & & & & \\
\hline 20 & 2014-01-03 7:00 & 16.2 & 12.5 & 9.8 & 12.6 & 11 & 10.3 & 12.6 & 10.2 & 14.7 & 16.4 & 15 & 17.3 & 18.2 \\
\hline 21 & 2014-01-03 10:00 & 15.3 & 14.6 & 19.4 & 17.4 & 13 & 16.3 & 16.3 & 12.9 & 17.1 & 20.6 & 19.8 & 19.6 & 20.8 \\
\hline 22 & $2014-01-03 \quad 13: 00$ & 20.8 & 17.9 & 25.2 & 19.4 & 19.6 & 23.6 & 22.8 & 21.7 & 20 & 22.6 & 20.4 & 21.1 & 23.4 \\
\hline 23 & 2014-01-03 16:00 & 24.9 & 24.5 & 23.4 & 22.6 & 21.1 & 22.3 & 22.6 & 22.2 & 20.4 & 20.9 & 22 & 19.5 & 20.8 \\
\hline 24 & 2014-01-03 19:00 & 19.3 & 19.7 & 17.4 & 19.6 & 17.1 & 19.6 & 19 & 15.3 & 14.4 & 17.4 & 16.2 & 18 & 17.7 \\
\hline 25 & $2014-01-0322: 00$ & 16.4 & 16 & 15 & 18.8 & 16.4 & 17.2 & 16.3 & 13.2 & 11.8 & 15.1 & 14.3 & 16.5 & $17.1 \nabla$ \\
\hline & Data_T & p43_Origi & & & & & & & 1 & & & & & $\nabla$ \\
\hline Reac & & & & & & & & & & & 囲 & $\amalg$ & 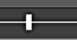 & $+\quad 100 \%$ \\
\hline
\end{tabular}

Hình 2. Dũ liệu nhiệt độ thu thập được tại 43 trạm quan trắc 3h. 
Như đã trình bày trong phần 1 , có rất nhiều yêu cầu cần phải thực hiện cho bước chuẩn hóa dữ liệu, tuy nhiên trong nội dung của bài báo, nhóm tác giả chỉ tập trung vào phát hiện và xử lý các ngoại lai cho dữ liệu nhiệt độ tại 43 trạm này. Trong phần 3 dưới đây, sẽ trình bày những nội dung cơ bản về phát hiện và xử lý ngoại lai, trong đó có 2 phương pháp được sử dụng để phát hiện ngoại lai cho dữ liệu có số chiều thấp là $\mathrm{Z}$ - Score và Box - plot. Đây cũng là 2 phương pháp mà nhóm tác giả sử dụng cho việc phát hiện ngoại lai trong tệp dữ liệu nhiệt độ ở trên.

\section{Phát hiện và xử lý ngoại lai}

\subsection{Giói thiệu về dũ̃ liệu ngoại lai}

Một điểm ngoại lai là một điểm dữ liệu khác biệt đáng kể so với phần còn lại của tập dữ liệu (C. Aggarwal, 2017). Các giá trị ngoại lai thường được xem như là các mẫu dữ liệu đặc biệt, cách xa khỏi phần lớn dữ liệu khác trong tập dữ liệu (N.N.R Ranga Suri et al., 2018).

Hình 3a thể hiện tập dữ liệu nhiệt độ quan trắc được của trạm 48855 - Đà Nẵng, dữ liệu này không chứa giá trị ngoại lai. Hình $3 \mathrm{~b}$ thể hiện dữ liệu nhiệt độ quan trắc của trạm 48918 - Côn Đảo, dữ liệu này có chứa một số giá trị ngoại lai. Các điểm dữ liệu này cách xa khỏi phần lớn các phần tử khác trong tập dữ liệu đã được chỉ ra cụ thể trong hình.

Có rất nhiều nguyên nhân chủ quan và khách quan dẫn tới sự xuất hiện của các điểm ngoại lai
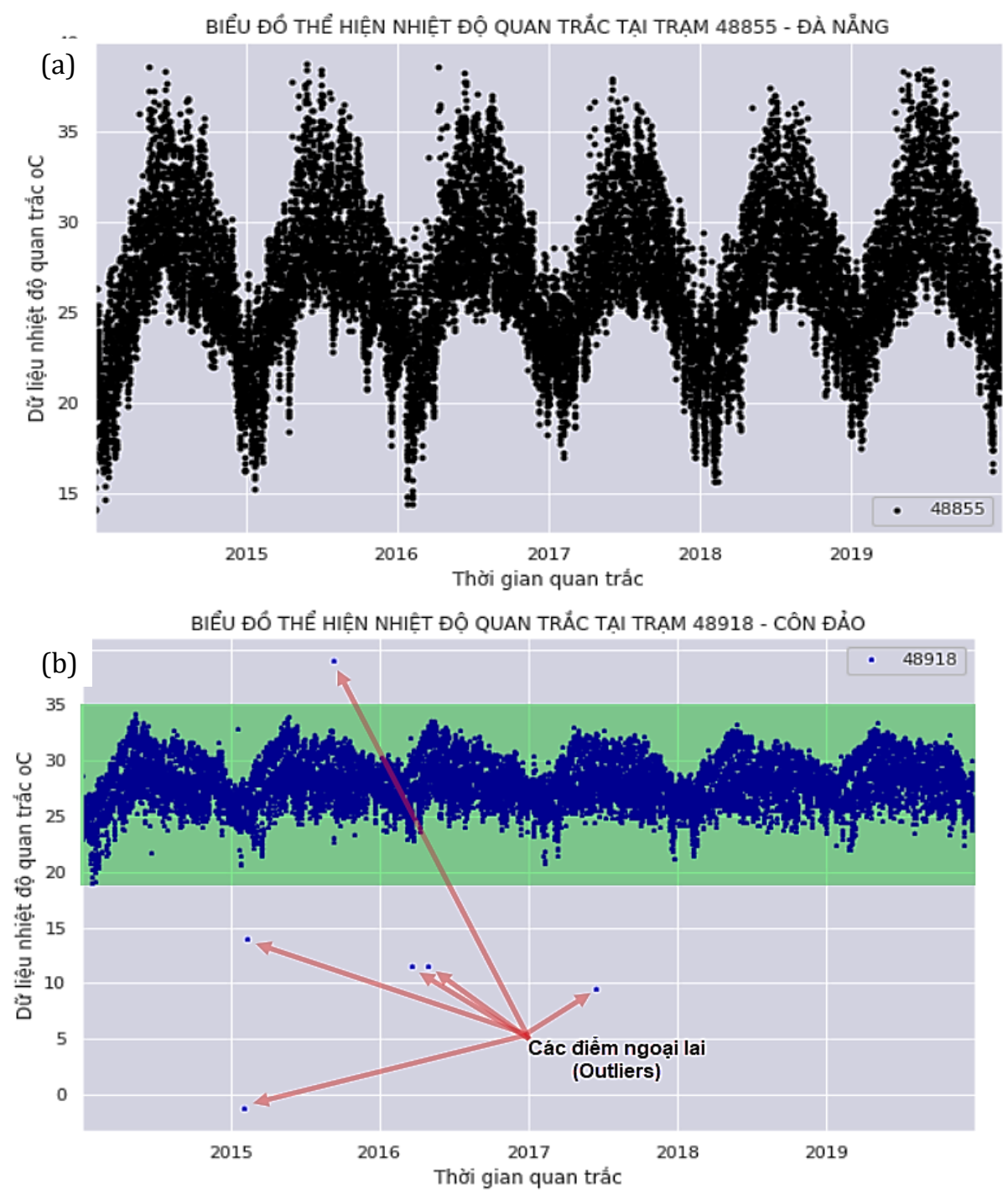

Hình 3. (a) Minh họa tập dũ liệu không chứa dũ liệu ngoại lai; (b) Minh họa tập dũ liệu chứa các điểm dũ liệu ngoại lai. 
trong tập dữ liệu như: Các lỗi nhập dữ liệu do con người gây ra; Các lỗi đo lường do thiết bị, dụng cụ lấy mẫu, thí nghiệm gây ra; Do cố ý tạo ra để phục vụ việc kiểm tra các phương pháp phát hiện; Các lối xử lý dữ liệu phát sinh trong quá trình thao tác dữ liệu; Các lỗi do lấy mẫu được trích xuất hoặc trộn dữ liệu từ các nguồn sai khác nhau; Do tự nhiên gây ra, đây không phải là lỗi mà là các giá trị quan sát thật tuy nhiên rất hiếm khi xuất hiện (N.N.R Ranga Suri et al., 2018).

Trong khai phá dữ liệu và trong các tài liệu thống kê, dữ liệu ngoại lai còn được gọi là dữ liệu bất thường (anomalies), lệch lạc (deviants),... Trong hầu hết các ứng dụng, dữ liệu được tạo ra bởi quá trình sinh dữ liệu, phản ánh hoạt động của hệ thống hoặc các quan sát thu thập về các thực thể. Khi quá trình tạo ra có những vấn đề bất thường, kết quả sẽ tạo ra các ngoại lai. Do đó, các giá trị ngoại lai thường chứa đựng những thông tin hữu ích về những đặc điểm bất thường của hệ thống và thực thể ảnh hưởng tới quá trình sinh dữ liệu. Việc phát hiện dữ liệu bất thường giúp chúng ta có những hiểu biết sâu sắc về từng ứng dụng cụ thể. Một số ứng dụng của dữ liệu ngoại lai trong thực tế có thể chỉ ra như (C. Aggarwal, 2017):

- Hệ thống phát hiện xâm nhập (Intrusion detection systems)

- Phát hiện gian lận tín dụng (Credit card fraud)

- Các sự kiện cảm biến quan tâm (Interesing sensor events)

- Trong chuẩn đoán y tế (Medical diagnosis)

- Trong thực thi pháp luật (Law enforcement)

- Trong khoa học trái đất (Earth science)

Có nhiều phương pháp để phát hiện các điểm dữ liệu ngoại lại, tác giả C. Aggarwal (2017) đã liệt kê một số phương pháp cơ bản được sử dụng bao gồm:

- Phân tích giá trị cực trị (Extreme Value Analysis): Đây là phương pháp cơ bản nhất được sử dụng để phát hiện các điểm ngoại lai, áp dụng tốt cho dữ liệu một chiều.

- Các mô hình xác suất và thống kê (Probabilistic and Statistical Models): Phương pháp này áp đặt một phân bố cụ thể trên tập dữ liệu như phân bố đều, phân bố Bernoulli, phân bố Poisson,... Sau đó, tính xác suất cho các phần tử thuộc tập dữ liệu ban đầu, các phần tử nào có xác suất thấp sẽ được cho là điểm ngoại lai.

- Các mô hình tuyến tính (Linear Models): Với phương pháp này, sẽ phải chuyển đổi tập dữ liệu ban đầu sang không gian ít chiều hơn bằng cách sử dụng tương quan tuyến tính. Sau đó, khoảng cách của từng điểm dữ liệu đến mặt phẳng ở không gian mới sẽ được tính toán và khoảng cách này sẽ được dùng để tìm ra các điểm ngoại lai.

- Các mô hình dựa trên lân cận (Proximity based Models): Phương pháp này dựa trên ý tưởng là mô hình hóa các điểm ngoại lai sao cho chúng hoàn toàn tách biệt khỏi toàn bộ các điểm dữ liệu còn lại. Phân cụm, phân tích dựa trên mật độ, phân tích dựa trên người hàng xóm gần nhất là các hướng tiếp cận chính của phương pháp này.

- Các mô hình dựa trên lý thuyết thông tin (Information Theoretic Models): Phương pháp này dựa trên nguyên lý các điểm ngoại lai sẽ làm tăng giá trị minimum code length khi mô tả tập dữ liệu.

Dữ liệu nhiệt độ thu thập được từ các trạm quan trắc đều là các dữ liệu một chiều. Quá trình làm việc với dữ liệu này, có 2 dạng ngoại lai chủ yếu được phát hiện và xử lý bao gồm:

- Ngoại lai trái (Left outlier): Là các điểm ngoại lai có giá trị cực tiểu (Extreamly low) trong tập mẫu quan sát (C. Aggarwal, 2017).

- Ngoại lai phải (Right outlier): Là các điểm ngoại lai có giá trị cực đại (Extreamly large) trong tập mẫu quan sát (C. Aggarwal, 2017).

Do đặc điểm của tập dữ liệu, phương pháp được dùng để phát hiện dữ liệu ngoại lai áp dụng cho 43 trạm quan trắc của Việt Nam thuộc nhóm đầu tiên đã chỉ ra ở trên là phân tích giá trị cực trị, trong đó 2 phương pháp chính là sử dụng Z- Score và sử dụng đồ thị Box - plot. Chi tiết của hai phương pháp này được trình bày trong phần 3.2 dưới đây.

\subsection{Phát hiện ngoại lai cho dũ liệu một chiều}

\subsubsection{Phương pháp sử dụng $Z$ - Score}

Điểm tiêu chuẩn hay Z - Score chỉ ra một thành phần chênh lệch so với trung bình là bao nhiêu độ lệch chuẩn (C. Aggarwal, 2017). Z - Score của bất kỳ một điểm dữ liệu nào được tính theo công thức:

$$
z=\frac{(x-\mu)}{\sigma}
$$

Trong đó: $x$ là giá trị của điểm dữ liệu cần tính $Z$ - Score; $\mu$ là giá trị trung bình của tập dữ liệu; $\sigma$ là độ lệch chuẩn của tập dữ liệu. (Nếu $\mathrm{z}<0$ thể hiện 
điểm dữ liệu đó nhỏ hơn giá trị trung bình; Nếu $z>0$ thể hiện điểm dữ liệu đó lớn hơn giá trị trung bình; Nếu $z=0$ thể hiện điểm dữ liệu đó bằng với giá trị trung bình).

Sau khi tính toán Z - Score cho từng điểm trong tập dữ liệu, một ngưỡng (threshold) sẽ được thiết lập để lọc các điểm này so với giá trị trung bình. Nếu tập dữ liệu theo phân phối chuẩn như chỉ ra trong Hình 4, cho thấy:

- Với ngưỡng 2,5 (-2.5<Z - Score $<+2.5)$ có $99 \%$ điểm dữ liệu nằm trong phạm vi 2,5 lần độ lệch chuẩn.

- Với ngưỡng 3,0 ( $-3.0<Z$ - Score $<+3.0)$ có $99,8 \%$ điểm dữ liệu nằm trong phạm vi 3,0 lần độ lệch chuẩn.

- Với ngưỡng 5,0 $(-5.0<Z$ - Score $<+5.0)$ có $99,9999426 \%$ điểm dữ liệu nằm trong phạm vi 5,0 lần độ lệch chuẩn.

Như vậy, bằng cách gắn thẻ, hoặc lọc các điểm dữ liệu nằm ngoài ngưỡng nhất định, chúng ta có thể phân loại các điểm dữ liệu thành ngoại lai và không ngoại lai. Z - Score là một phương pháp đơn giản nhưng khá mạnh mẽ để phát hiện các điểm ngoại lai trong một tập dữ liệu. Tuy nhiên, phương pháp này chỉ tốt đối với dữ liệu có số chiều thấp và có phân phối chuẩn.

\subsubsection{Phương pháp sử dụng biểu đồ Box - plot}

Biểu đồ Box - plot được sử dụng để đo khuynh hướng phân tán và xác định các giá trị ngoại lai của tập dữ liệu. Biểu đồ Box - plot chia tập dữ liệu thành các khoảng phần tư, phần thân của biểu đồ bao gồm một chiếc hộp, biểu đồ thể hiện 5 giá trị của tập dữ liệu (Hình 5) bao gồm:

- Giá trị bé nhất (Minimum) của tập dữ liệu được xác định bằng Q1 - 1.5 * IQR;

- Tứ phân vị thứ nhất (Q1) của tập dữ liệu.

- Tứ phân vị thứ hai $(\mathrm{Q} 2)$ chính là giá trị trung vị (Median) của tập dữ liệu.

- Tứ phân vị thứ ba (Q3) của tập dữ liệu.

- Giá trị lớn nhất (Maximum) của tập dữ liệu có giá trị bằng $\mathrm{Q} 3+1.5^{*} \mathrm{IQR}$.

Nếu tập dữ liệu có chứa các giá trị ngoại lai thì chiều dài tối đa của 2 râu tính từ mỗi cạnh hộp sẽ

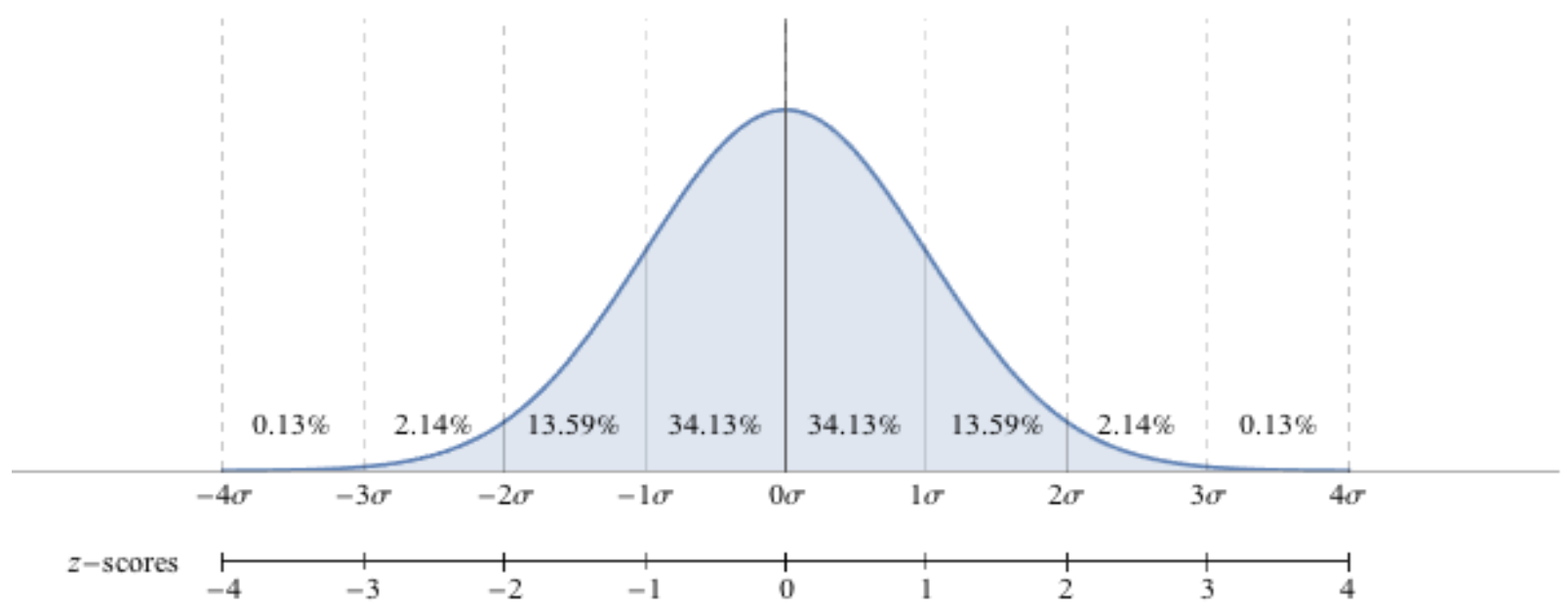

Hình 4. Tỷ lệ điểm dũ liệu nằm trong phạm vi theo ngưỡng Z - Score với phân phối chuẩn.

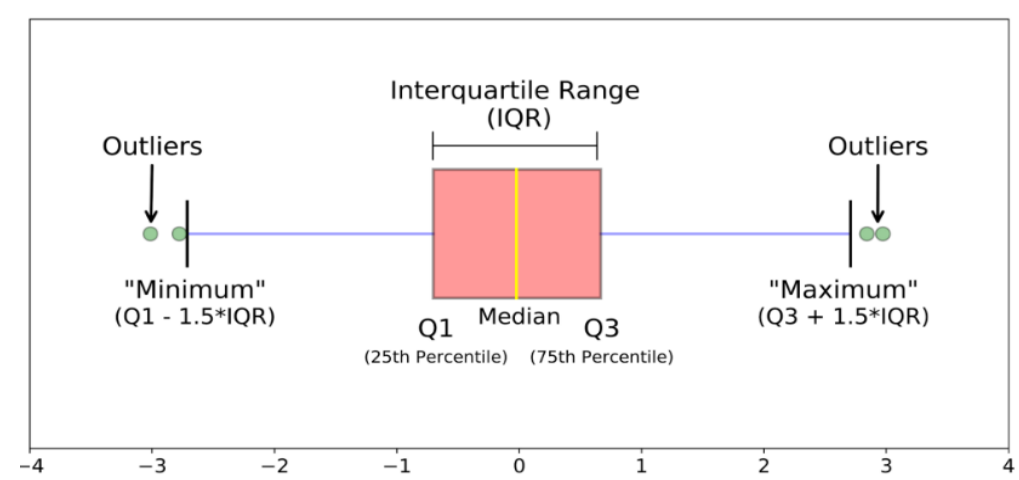

Hình 5. Hình dạng và các giá trị của tập dũ liệu thể hiện trên biểu đồ Box - plot. 
được xác định bằng 1,5 lần độ trải giữa (IQR Interquatile Range). Các điểm dữ liệu nằm ngoài râu Minimum được xem xét là các điểm ngoại lai trái (Left outlier), các điểm dữ liệu nằm ngoài râu Maximum được xem xét là các điểm ngoại lai phải (Right outlier). Các điểm dữ liệu ngoại lai này được thể hiện bằng dấu chấm tròn trên biểu đồ Box - plot. Như trong hình 5 , thể hiện 2 điểm ngoại lai trái và 2 điểm ngoại lai phải. Ngoài ra, biểu đồ Box - plot còn cung cấp thông tin về hình dạng của tập dữ liệu. Nếu đường trung vị (Median) chia hộp thành 2 nửa đều nhau, thì tập dữ liệu này đối xứng; Nếu nửa phải lớn hơn nửa trái thì tập dữ liệu bị lệch phải và ngược lại, nếu nửa trái lớn hơn nửa phải thì tập dữ liệu bị lệch trái (Munzer, 2014).

Box - plot là đồ thị trực quan thường được các nhà phân tích, thống kê, nhà khoa học dữ liệu sử dụng để tóm tắt thông tin về một biến dữ liệu định lượng bất kỳ phục vụ cho nhiều giai đoạn trong quá trình khai thác và tiền xử lý dữ liệu (Nguyễn Văn Tuấn, 2014).

\subsection{Xử lý dũ̃ liệu ngoại lai}

Việc phát hiện các điểm dữ liệu ngoại lai có thể thực hiện bằng nhiều phương pháp khác nhau, sau khi phát hiện được các điểm ngoại lai yêu cầu đặt ra là phải xử lý chúng. Các điểm dữ liệu ngoại lai có ảnh hưởng rất lớn đến độ chính xác của các mô hình, việc lựa chọn được phương pháp nào để xử lý sao cho phù hợp với từng loại dữ liệu cụ thể thường khó hơn rất nhiều so với việc phát hiện ra chúng (N.N.R Ranga Suri et al., 2018).

Cũng tương tự như việc phát hiện, để xử lý các điểm ngoại lai cũng có nhiều phương pháp. Mỗi một phương pháp lại có ưu và nhược điểm riêng, việc chọn phương pháp xử lý nào tùy thuộc vào yêu cầu phân tích dữ liệu của từng bài toán cụ thể đặt ra. Dưới đây là tổng hợp các phương pháp xử lý ngoại lai chung cho tập dữ liệu:

- Loại bỏ các dòng chứa ngoại lai khỏi tập dữ liệu: Đây là cách xử lý ngoại lai đơn giản và dễ thực hiện nhất. Sau khi phát hiện các điểm ngoại lai, thực hiện xóa các dòng dữ liệu chứa giá trị ngoại lai này khỏi tập dữ liệu. Tuy nhiên, phương pháp này chỉ áp dụng cho tập dữ liệu chứa các biến độc lập. Với dữ liệu dạng chuỗi thời gian (Time series data), chúng ta không thể sử dụng phương pháp này để loại bỏ một điểm ngoại lai tại một vị trí vì các điểm dữ liệu trong chuỗi thời gian có mối quan hệ tương quan với nhau. Ngoài ra, với dữ liệu có nhiều thuộc tính khác nhau, nếu xóa cả dòng dữ liệu chứa một thuộc tính có giá trị ngoại lai sẽ làm mất thông tin trên các cột khác nếu cột này cần cho phân tích.

- Thay thế bằng một giá trị khác: Thay thế giá trị của các điểm ngoại lai bằng một giá trị khác phù hợp hơn với tập dữ liệu. Với phương pháp này vấn đề khó khăn gặp phải đó là lựa chọn giá trị nào để thay thế cho giá trị của điểm ngoại lai? Câu trả lời là tùy thuộc vào từng loại dữ liệu, kiểu dữ liệu và trong những ngữ cảnh cụ thể để xác định được giá trị thay thế phù hợp nhất. Trong một số trường hợp có thể thay thế các giá trị ngoại lại bằng giá trị trung bình (mean) của tập dữ liệu, hoặc thay thế bằng một giá trị cụ thể (specific value) do các nhà phân tích dữ liệu, chuyên gia đề xuất.

- Thay thế giá trị của các điểm ngoại lai bằng NULL (empty): Việc thực hiện này sẽ chuyển đổi các điểm ngoại lai thành các điểm thiếu dữ liệu (missing value). Các điểm ngoại lai bây giờ được xem xét như là một điểm dữ liệu thiếu trong tập dữ liệu để xử lý.

Không có một phương pháp, cách thức xử lý ngoại lai chung nào áp dụng cho tất cả các bài toán, các kiểu dữ liệu khác nhau (N.N.R Ranga Suri et al., 2018). Vì vậy, để lựa chọn được phương pháp phù hợp cần có những hiểu biết sâu sắc về tập dữ liệu, về bài toán đang giải quyết, có thể sử dụng chỉ một phương pháp xử lý ngoại lai và/hoặc kết hợp cả 3 nhóm phương pháp đã chỉ ra ở trên để xử lý ngoại lai cho cùng một tập dữ liệu.

\section{4. Áp dụng cho dữ liệu nhiệt độ tại các trạm quan trắc $3 \mathrm{~h}$ của Việt Nam}

Tại mục 2 và 3 của bài báo, tác giả đã trình bày về tập dữ liệu nhiệt độ thu thập được từ 43 trạm quan trắc 3 h của Việt Nam, hai phương pháp được sử dụng phổ biến và hiệu quả trong việc phát hiện ngoại lai cho dữ liệu có số chiều thấp là $\mathrm{Z}$ - Score và Box - plot. Ở phần này, nhóm tác giả sẽ sử dụng kỹ năng lập trình kết hợp với các thư viện, hệ thống mã nguồn mở để áp dụng các phương pháp đó cho việc phát hiện và xử lý ngoại lai với dữ liệu nhiệt độ tại 43 trạm quan trắc 3 h của Việt Nam.

Dữ liệu nhiệt độ phụ thuộc rất lớn vào vị trí địa lý, với các tỉnh biên giới phía bắc nước ta nhiệt độ có thể hạ thấp xuống $0^{\circ} \mathrm{C}$ hoặc thậm chí là âm vẫn có thể coi là bình thường. Tuy nhiên, với các tỉnh phía Nam, nếu dữ liệu nhiệt độ ghi nhận được có 
giá trị thấp dưới $15^{\circ} \mathrm{C}$ có thể xem xét nó là các điểm ngoại lai cần phải được kiểm tra và xử lý. Do vậy, việc phát hiện và xử lý dữ liệu ngoại lai được thực hiện lần lượt cho từng trạm và không có một ngưỡng chung nào được áp dụng cho tất cả các tram.

Trong khuôn khổ của bài báo, nhóm tác giả chọn một trạm điển hình (Trạm 48918: Côn Đảo) trong số 43 trạm để trình bày, minh họa chi tiết việc phát hiện và xử lý các điểm ngoại lai. Các trạm còn lại cũng sẽ được xử lý lần lượt theo các bước tương tự như với trạm này. Nhóm tác giả lựa chọn trạm 48918 trình bày trong bài báo vì đây là trạm nằm trên đảo có điều kiện khí hậu khắc nghiệt, việc truyền dữ liệu gặp nhiều khó khăn,... Có nhiều nguyên nhân dần đến các điểm ngoại lai trong dữ liệu quan trắc. Trạm 48918 có số thứ tự 39 trong Bảng 1 , vị trí của trạm này được thể hiện tương đối trong Hình 1 ở trên và chi tiết trong Hình 6.

Nhóm tác giả lựa chọn ngôn ngữ lập trình Python, mã nguồn được viết trên hệ thống Google Colab, sử dụng 3 thư viện nguồn mở để tính toán và trực quan hóa bao gồm: Pandas, Matplotlib,
Seaborn. Tiến hành đọc và trích xuất dữ liệu quan trắc của trạm 48918 trong tập dữ liệu thô Data_Temp43_Original.csv. Bảng 2 chỉ ra thông số của tập dữ liệu và Hình 7 thể hiện biểu đồ tần suất (histogram) của dữ liệu nhiệt độ trạm 48918.

Bảng 2. Thông số tập dũ liệu quan trắc của trạm 48918.

\begin{tabular}{|c|c|c|}
\hline TT & Thời điểm bắt đầu dũ̃ liệu & \begin{tabular}{|c|}
$01: 00: 0001$ \\
$01-2014$
\end{tabular} \\
\hline 1 & Thời điểm kết thúc dữ liệu & \begin{tabular}{|c|}
$22: 00: 0031$ \\
$12-2019$
\end{tabular} \\
\hline 2 & Tổng số điểm dữ liệu & 17528 \\
\hline 3 & Số điểm có dữ liệu & 17495 \\
\hline 4 & Số điểm dữ liệu thiếu & 33 \\
\hline 5 & Giá trị trung bình của tập dữ liệu & 27.8478 \\
\hline 6 & Độ lệch chuẩn của tập dữ liệu & 2.0407 \\
\hline 7 & Giá trị cực tiểu & -1.3 \\
\hline 8 & Tứ phân vị thứ nhất (Q1) & 26.4 \\
\hline 9 & Tứ phân vị thứ hai (Q2) & 27.8 \\
\hline 10 & Tứ phân vị thứ ba (Q3) & 29.1 \\
\hline 11 & Giá trị cực đại & 39.0 \\
\hline
\end{tabular}

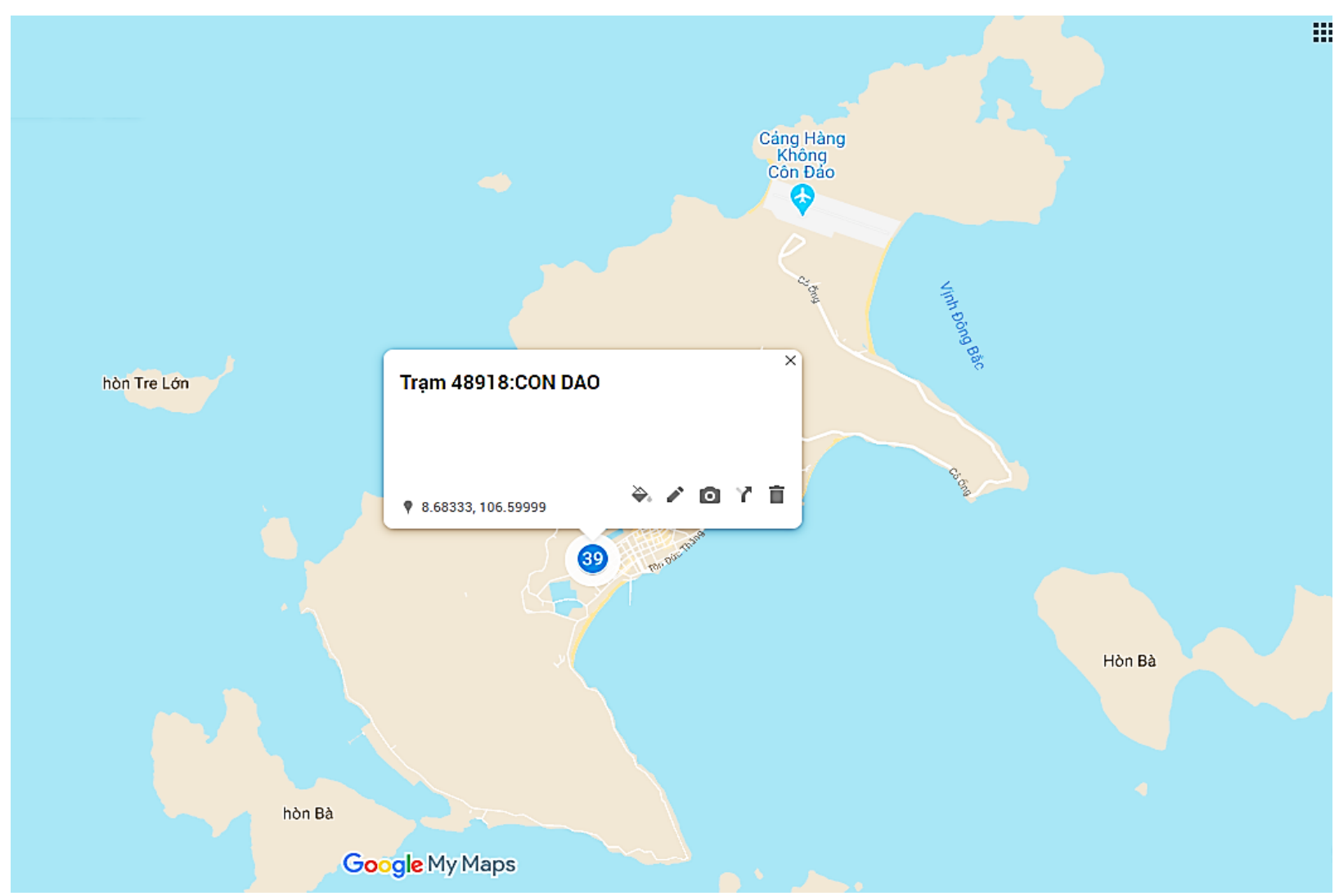

Hình 6. Vị trí trạm 48918: CON DAO trên Google Maps. 


\subsection{Sử dụng $Z$ - Score phát hiện ngoại lai trong dữ liệu nhiệt độ của trạm 48918}

Thực hiện tính giá trị Z - Score theo công thức (1) đã trình bày trong phần 3.2.1 của tất cả các điểm trong tập dữ liệu trạm 48918. Mã lập trình việc tính toán và kết quả được thể hiện như trong Hình 8.

Để xem xét và xác định ngoại lai, sau khi tính được giá trị $Z$ - score đó là phải chọn một ngưỡng (threshold) phù hợp. Khi trao đổi với chuyên gia khí tượng thủy văn, cùng với các số liệu thống kê nhiệt độ tại khu vực phía Nam nói chung, Côn Đảo nói riêng thì nhiệt độ ở đây nằm trong khoảng $1^{\circ} \mathrm{C} \div 38^{\circ} \mathrm{C}$. Như vậy các thông số quan trắc thấp hơn $17^{\circ} \mathrm{C}$ và cao hơn $38^{\circ} \mathrm{C}$ sẽ được xem xét là ngoại lai.

Theo như Bảng 3, với ngưỡng $Z=5$, thỏa mãn điều kiện giới hạn nhiệt độ trong khoảng $17^{\circ} \mathrm{C} \div$ $38^{\circ} \mathrm{C}$. Vì vậy, giá trị 5 được chọn là ngưỡng để lọc các điểm xem xét ngoại lai. Kết quả lọc các điểm có $\mathrm{Z}$ - Score nằm ngoài ngưỡng 5 cho trạm 48918 như trong Hình 9.

Như vậy, theo phương pháp $Z$ - Score với ngưỡng lọc chọn bằng 5 có tất cả 6 điểm dữ liệu được xem xét là ngoại lai, trong đó có 5 điểm ngoại lai trái (zscore < 0) và 1 điểm ngoại lai phải (zscore $>0$ ).

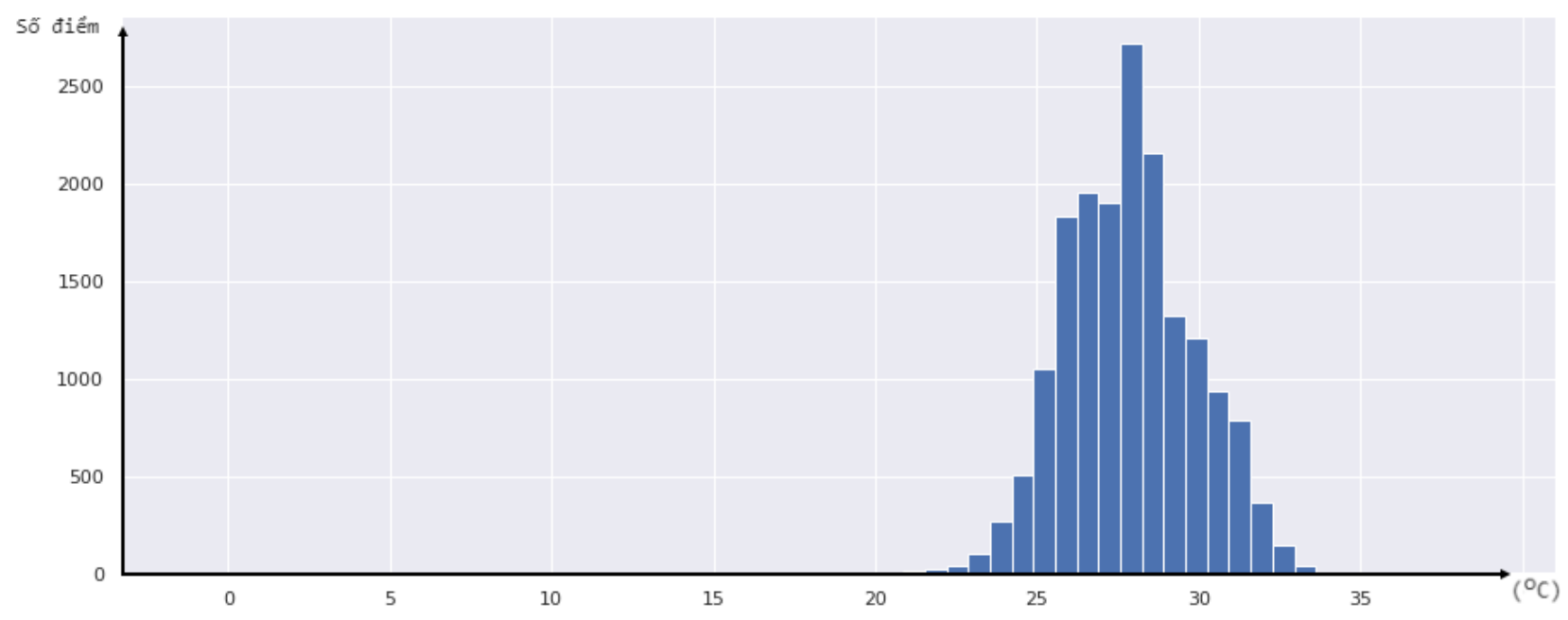

Hình 7. Biểu đồ histogram của tập dũ liệu nhiệt độ trạm 48918.

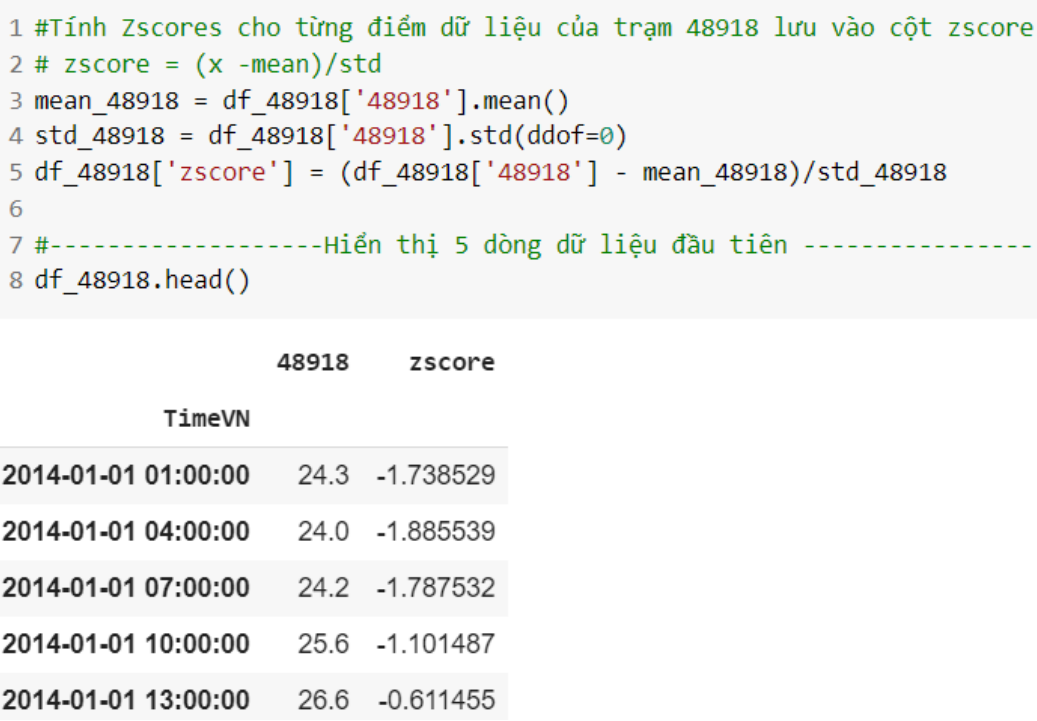

Hình 8. Kết quả tính Z - Score cho các điểm quan trắc của trạm 48918. 
Bảng 3. Ngương và khoảng nhiệt tương úng với ngương thiết lập của trạm 48918.

\begin{tabular}{|c|c|c|c|}
\hline TT & $\begin{array}{c}\text { Ngưỡng } \\
\text { (threshold) }\end{array}$ & $\begin{array}{c}\text { Giới hạn theo } \\
\text { ngưỡng Z }\end{array}$ & $\begin{array}{c}\text { Khoảng nhiệt độ nằm } \\
\text { trong giới hạn } \\
\text { ngưỡng Z (mean: } \\
27.85 \mid \text { std:2.04) }\end{array}$ \\
\hline 1 & 3.0 & $\begin{array}{c}-3,0<=\mathrm{Z}<= \\
3,0\end{array}$ & {$\left[21.73^{\circ} \mathrm{C}-33.97^{\circ} \mathrm{C}\right]$} \\
\hline 2 & 4.0 & $\begin{array}{c}-4,0<=\mathrm{Z}<= \\
4,0\end{array}$ & {$\left[19.69^{\circ} \mathrm{C}-36.01^{\circ} \mathrm{C}\right]$} \\
\hline 3 & 5.0 & $\begin{array}{r}-5,0<=\mathrm{Z}<= \\
5,0\end{array}$ & {$\left[17.65^{\circ} \mathrm{C}-38.05^{\circ} \mathrm{C}\right]$} \\
\hline 4 & 5.5 & $\begin{array}{r}-5,5<=\mathrm{Z}<= \\
5,5\end{array}$ & {$\left[16.63^{\circ} \mathrm{C}-39.07^{\circ} \mathrm{C}\right]$} \\
\hline
\end{tabular}

\subsection{Sử dụng biểu đồ Box - plot phát hiện ngoại lai trong dũ liệu nhiệt độ của trạm 48918}

Sử dụng thư viện Matplotlib và Seaborn để dựng biểu đồ Box - Plot cho dữ liệu nhiệt độ trạm 48918. Theo như biểu đồ Box - plot trong Hình $10 \mathrm{a}$, có thể nhận thấy có khá nhiều điểm dữ liệu nằm trên và dưới hai râu minimum và maximum của biểu đồ, về nguyên tắc các điểm này đều được xem xét là các điểm dữ liệu ngoại lai. Tuy nhiên, như đã trình bày, thông số nhiệt độ ở Côn Đảo thường nằm trong khoảng $17^{\circ} \mathrm{C} \div 38^{\circ} \mathrm{C}$. Do đó, từ biểu đồ Box - plot có thể lọc các điểm ngoại lai trái với ngưỡng $17^{\circ} \mathrm{C}$, các điểm ngoại lai phải với ngưỡng $38^{\circ} \mathrm{C}$.

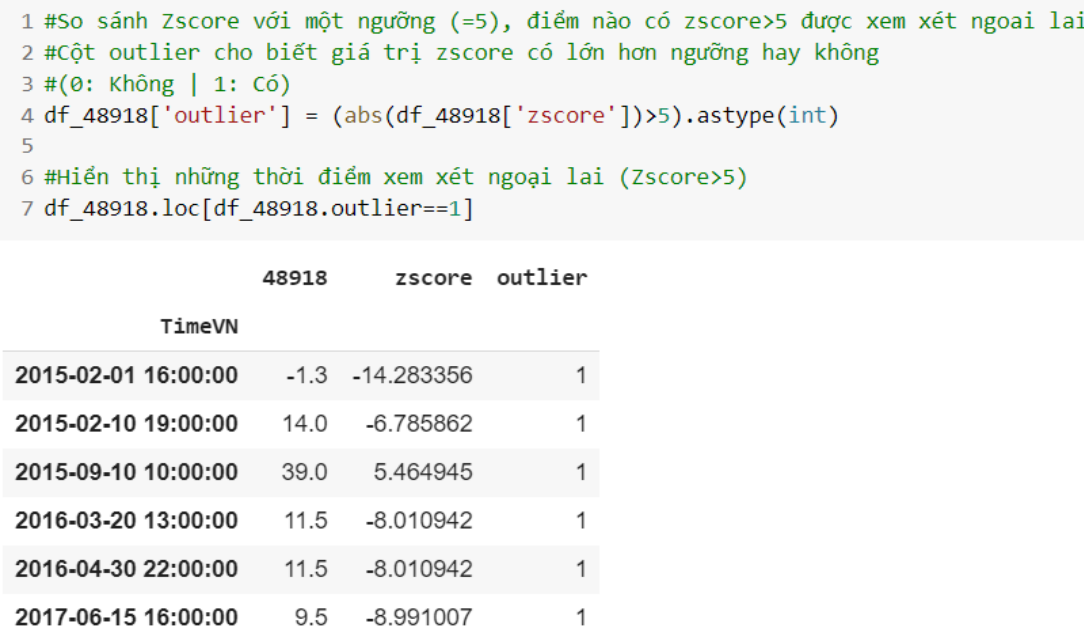

Hình 9. Phát hiện dũ liệu ngoại lai sử dụng Z - Score với trạm 48918.

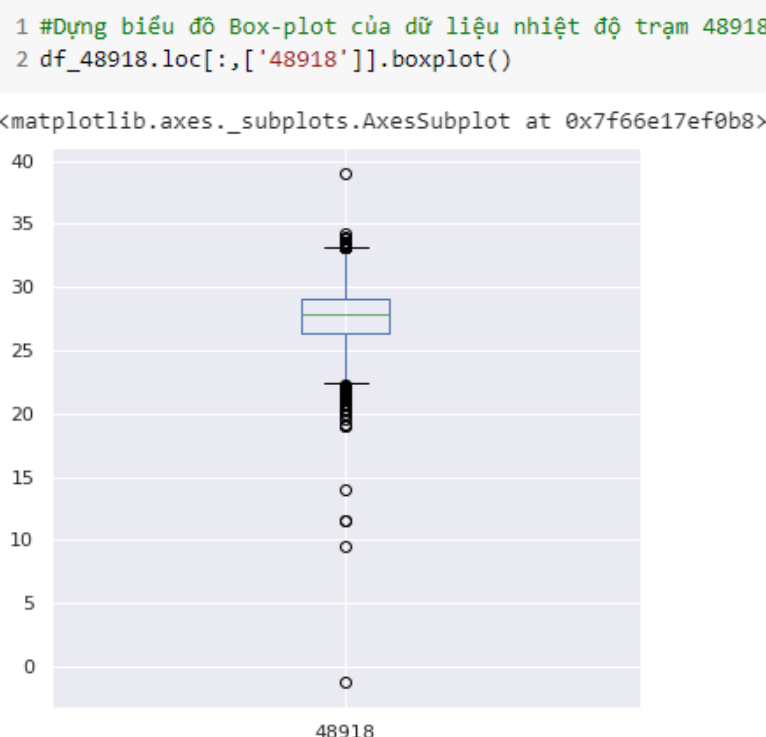

(a)

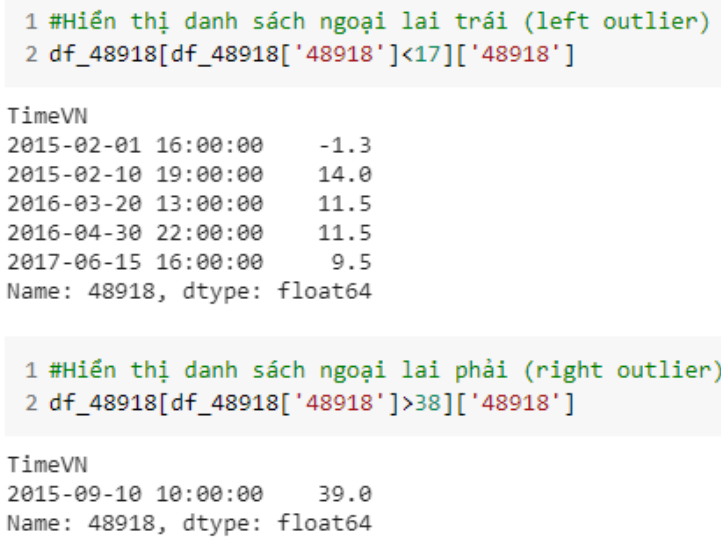

(b)

Hình 10. Biểu đồ box - plot và các điểm xem xét ngoại lai của trạm 48918. 
Kết quả tách các điểm ngoại lai trái - phải được thể hiện trong Hình 10b.

Theo như kết quả thu được cả hai phương pháp sử dụng Z - Score và Biểu đồ Box - plot đều cho cùng một danh sách các điểm ngoại lai với 6 điểm dữ liệu chi tiết như trong Bảng 4.

Bảng 4. Thò̀i điểm và giá trị quan trắc xem xét ngoại lai của trạm 48918.

\begin{tabular}{|c|c|c|c|}
\hline TT & Thời điểm & $\begin{array}{l}\text { Giá trị quan } \\
\text { trắc }\end{array}$ & $\begin{array}{c}\text { Loại ngoại } \\
\text { lai }\end{array}$ \\
\hline 1 & $2015-02-0116: 00: 00$ & -1.3 & \multirow{5}{*}{$\begin{array}{l}\text { Ngoại lai } \\
\text { trái (Left } \\
\text { outlier) }\end{array}$} \\
\hline 2 & 2015 - 02 - 10 19:00:00 & 14.0 & \\
\hline 3 & $2016-03-2013: 00: 00$ & 11.5 & \\
\hline 4 & $2016-04-3022: 00: 00$ & 11.5 & \\
\hline 5 & $2017-06$ - 15 16:00:00 & 9.5 & \\
\hline 6 & $2015-09-1010: 00: 00$ & 39.0 & \begin{tabular}{|} 
Ngoại lai \\
phải \\
(Right \\
outlier)
\end{tabular} \\
\hline
\end{tabular}

Đây chỉ là các điểm xem xét ngoại lai, để khẳng định có phải là ngoại lai thật hay không thì cần phải được kiểm chứng.

\subsection{Kiểm chúng các điểm ngoại lai phát hiện được}

Dữ liệu nhiệt độ thu nhận được từ các trạm quan trắc như đã trình bày có dạng chuỗi thời gian, sau mỗi khoảng thời gian $3 \mathrm{~h}$ sẽ có một điểm dữ liệu mới. Do vậy, để khẳng định đây là các điểm ngoại lai, cần xem xét điểm dữ liệu này trong một chuỗi dữ liệu tương ứng với điểm đó. Trong phần này, nhóm tác giả sẽ thực hiện kiểm chứng tại hai thời điểm có giá trị nhỏ nhất $(-1.3)$ và lớn nhất (39.0), các vị trí khác được kiểm chứng tương tự và mô tả cụ thể trong phần mã nguồn của bài báo (địa chỉ mã nguồn xử lý được cung cấp ở phần cuối của bài báo).

Với kết quả kiểm chứng dữ liệu nhiệt độ của trạm 48918 tại thời điểm 16h ngày 01/02/2015 như thể hiện trong Hình 11 , có thể khẳng định dữ liệu quan trắc thu thập được tại thời điểm này là hoàn toàn sai lệch. Nhiệt độ ghi nhận tại thời điểm 16h phải có mối tương quan với nhiệt độ tại thời điểm trước đó lúc $13 \mathrm{~h}$ và sau đó lúc $19 \mathrm{~h}$; ngoài ra, đồ thị biểu diễn nhiệt độ của trạm trong khoảng thời gian 5 ngày từ $1 \mathrm{~h}$ ngày $01 / 02 / 2015$ đến $22 \mathrm{~h}$ ngày $05 / 02 / 2015$ (Hình $11 b$ ) cũng thể hiện rõ mức độ sai khác dữ liệu tại thời điểm này.

Tương tự như vậy, Hình 12 thể hiện kết quả kiểm chứng dữ liệu quan trắc tại thời điểm $10 \mathrm{~h}$ ngày 10/09/2015. Hình 12a hiển thị toàn bộ số liệu quan trắc trong ngày 10/09/2015 tại các thời điểm $1 \mathrm{~h}, 4 \mathrm{~h}, 7 \mathrm{~h}, 10 \mathrm{~h}, 13 \mathrm{~h}, 16 \mathrm{~h}, 19 \mathrm{~h}$ và $22 \mathrm{~h}$. Hình $12 \mathrm{~b}$ thể hiện đồ thị nhiệt độ quan trắc trong khoảng thời gian 5 ngày từ $1 \mathrm{~h}$ ngày $10 / 09 / 2015$ đến 22h ngày 15/09/2015. Dễ dàng nhận thấy dữ liệu quan trắc tại thời điểm $10 \mathrm{~h}$ ngày $10 / 09$ có mức độ sai khác tương đối lớn so với mặt bằng chung của các điểm đo. Hơn nữa, nhiệt độ tại thời điểm $10 \mathrm{~h}$ có giá trị là $39^{\circ} \mathrm{C}$ cao hơn nhiệt độ lúc $7 \mathrm{~h}$ là $27.8^{\circ} \mathrm{C}$ (chênh lệch $+11.2^{\circ} \mathrm{C}$ ) và cao hơn nhiệt độ ghi nhận lúc $13 \mathrm{~h}$ là $30.7^{\circ} \mathrm{C}$ (chênh lệch $+8.3^{\circ} \mathrm{C}$ ).

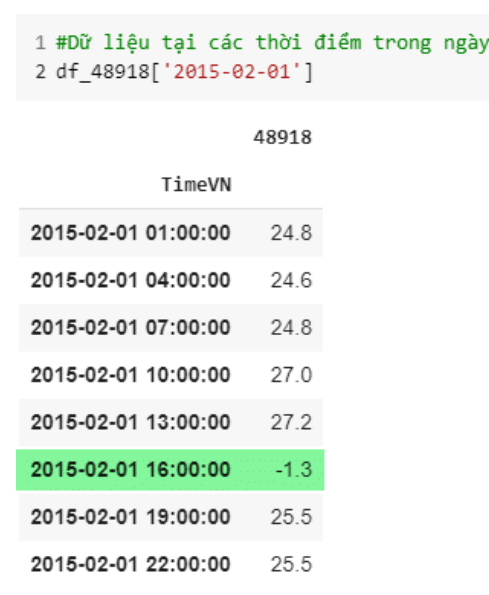

(a)

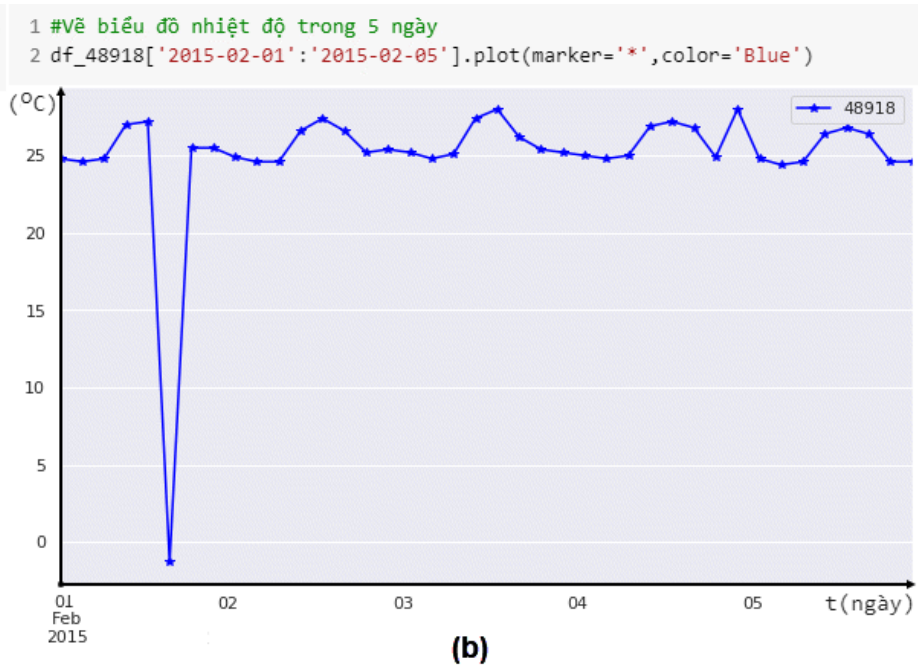

(b)

Hình 11. Kiểm chứng điểm ngoại lai trái có giá trị thấp nhất tại trạm 48918. 


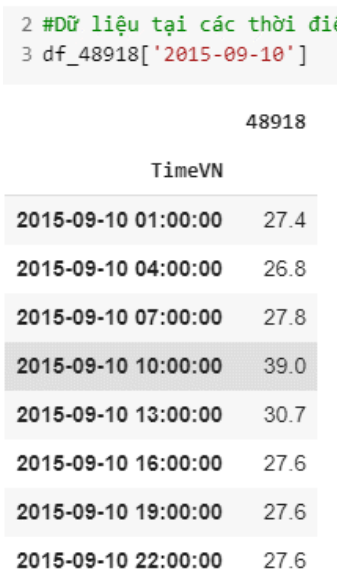

(a)

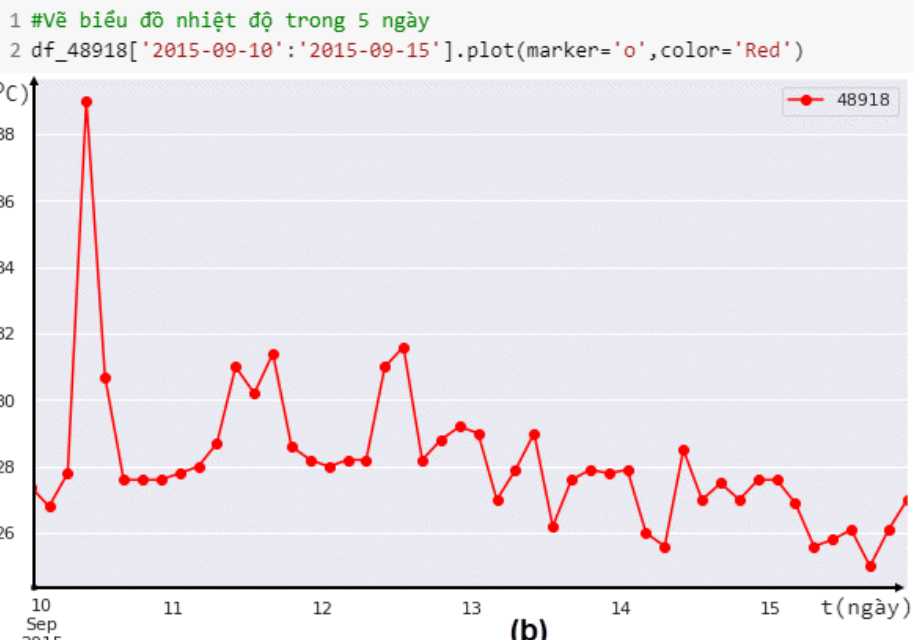

(b)

Hình 12. Kiểm chứng điểm ngoại lai phải có giá trị cao nhất tại trạm 48918.

Điều này trong thực tế là phí lý khi mức độ thay đổi trong khoảng $3 \mathrm{~h}$ là rất lớn và thời điểm nhiệt độ cao nhất trong ngày không phải là thời điểm $13 \mathrm{~h}$ như bình thường.

Từ các kết quả kiểm chứng có thể khẳng định các điểm này đều là các điểm dữ liệu ngoại lai, có giá trị sai khác rất lớn so với giá trị thực tế. Do vậy, dữ liệu tại các điểm này cần phải được xử lý trước khi sử dụng cho bất kỳ mục đích nào.

\subsection{Xử lý các điểm ngoại lai cho trạm 48918}

Trong phần 3.3, đã chỉ ra các phương pháp để xử lý ngoại lai nói chung, như đã trình bày dữ liệu nhiệt độ quan trắc thu thập được là dữ liệu dạng chuỗi thời gian, do vậy không thể sử dụng phương pháp loại bỏ các điểm này ra khỏi tập dữ liệu. Trong thực tế khi xử lý các điểm ngoại lai, nhóm tác giả chọn phương pháp thay thế các điểm ngọai lai về giá trị NULL (ứng với None trong Python Hình 13), xem các điểm ngoại lai là điểm dữ liệu thiếu (missing data) sau đó sẽ sử dụng phương pháp xử lý dữ liệu thiếu cho toàn bộ tập dữ liệu. Trong khuôn khổ nội dung của bài báo này, nhóm

\footnotetext{
1 \#Xử lý dữ liệu ngoại lai của trạm 48918

2 df_48918.10c['2015-02-01 16:00:00', ['48918']] = None

3 df_48918.10c['2015-02-10 19:00:00', ['48918']] = None

4 df_48918.10c['2016-03-20 13:00:00', ['48918']] = None

5 df_48918.10c['2016-04-30 22:00:00', ['48918']] = None

6 df_48918.10c ['2017-06-15 16:00:00', ['48918']] = None

7 df_48918.10c['2015-09-10 10:00:00', ['48918']] = None
}

Hình 13. Chuyển đổi các điểm ngoai lai về giá trị NULL để xử lý cho trạm 48918. tác giả không đề cập đến việc xử lý giá trị thiếu.

Quá trình phát hiện và xử lý ngoại lai tại 42 trạm còn lại được thực hiện lần lượt theo các bước như đã trình bày với trạm 48918.

\section{Kết luận}

Phát hiện và xử lý dữ liệu ngoại lai là yêu cầu bắt buộc và rất quan trọng trong quá trình chuẩn hóa dữ liệu. Các điểm ngoại lai có ảnh hưởng rất lớn tới độ chính xác của các mô hình dự đoán, dự báo. Nội dung bài báo này đã trình bày chi tiết về dữ liệu nhiệt độ thu thập được tại 43 trạm quan trắc 3h của Việt Nam; tổng quan về dữ liệu ngoại lai nói chung và hai phương pháp xử lý để phát hiện các điểm ngoại lai với dữ liệu có số chiều thấp là Z - Score và Box - plot. Kết quả chính của bài báo thể hiện ở phần thực nghiệm, áp dụng các phương pháp Z - Score và Box - plot để phát hiện các điểm ngoại lai cho một trạm điển hình đó là trạm 48918 - Côn Đảo. Các điểm ngoại lai sau khi phát hiện sẽ được kiểm chứng để sàng lọc một cách chính xác và xử lý về dạng dữ liệu thiếu (missing data). Sau khi thực hiện với toàn toàn bộ dữ liệu của các trạm, đã thu được một tập dữ liệu mới đã xử lý ngoại lai. Tập dữ liệu này tiếp tục được làm sạch với các yêu cầu khác như xử lý giá trị thiếu, chuẩn hóa,... và sẽ được sử dụng làm dữ liệu đầu vào cho các mô hình dự báo liển quan.

\section{Lò̀i cảm ơn}

Nghiên cứu này được hỗ trợ bởi đề tài "Nghiên cứu cơ sở khoa học và giải pháp ứng dụng trí tuệ nhân tạo để nhận dạng, hỗ trợ dự báo và cảnh báo 
một số hiện tượng khí tượng thủy văn nguy hiểm trong bối cảnh biến đổi khí hậu tại Việt Nam", mã số BĐKH.34/16 - 20."

\section{Tài liệu tham khảo}

Charu C., Aggarwal, (2017). Outlier Analysis, Springer International Publishing AG, New York.

Davy Cielen, Arno D. B., Meysman, Mohamed Ali, (2016). Introducing Data Science, Manning Publications Co.
Hermine N., Akouemo, Richard J. Povinelli, (2014). Time series outlier detection and imputation, IEEE.

Nguyễn Văn Tuấn, (2014). Phân tích dữ liệu với R, Nhà xuất bản tổng hợp Thành phố Hồ Chí Minh.

Ranga Suri, N. N. R, Narasimha Murty M., Athithan, G., (2018). Outlier Detection: Techniques and Applications, Springer Nature Switzerland AG, Cham.

Tamara Munzer, (2014). Visualization Analysis and Design,CRC Press. 